\title{
Knowledge and use of antibiotics in six ethnic groups: the HELIUS study
}

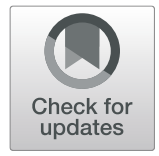

\author{
Emelie C. Schuts ${ }^{1 \dagger}$, Eline van Dulm ${ }^{2 *} \mathbb{D}$, Anders Boyd ${ }^{2,3}$, Marieke B. Snijder ${ }^{4,5}$, Suzanne E. Geerlings ${ }^{1}$, \\ Maria Prins ${ }^{1,2}$ and Jan M. Prins ${ }^{1}$
}

\begin{abstract}
Background: The increase of antimicrobial resistance, mainly due to increased antibiotic use, is worrying. Preliminary evidence suggests that antibiotic use differs across ethnic groups in the Netherlands, with higher use in people of non-Dutch origin. We aimed to determine whether appropriate knowledge and use of antibiotics differ by ethnicity and whether knowledge on antibiotics is associated with antibiotic use.

Methods: We performed a cross-sectional study analyzing baseline data (2011-2015) from a population-based cohort (HELIUS study), which were linked to data from a health insurance register. We included 21,617 HELIUS participants of South-Asian Surinamese, African-Surinamese, Turkish, Moroccan, Ghanaian, and Dutch origin. Fifteen thousand seven participants had available prescription data from the Achmea Health Data-base (AHD) in the year prior to their HELIUS study visit. Participants were asked five questions on antibiotic treatment during influenza-like illness, pneumonia, fever, sore throat and bronchitis, from which higher versus lower antibiotic knowledge level was determined. Number of antibiotic prescriptions in the year prior to the HELIUS study visit was used to determine antibiotic use.
\end{abstract}

Results: The percentage of individuals with a higher level of antibiotic knowledge was lower among all ethnic minority groups (range 57 to 70\%) compared to Dutch (80\%). After correcting for baseline characteristics, including medical conditions, first-generation African Surinamese and Turkish migrants received a significantly lower number of antibiotic prescriptions compared to individuals of Dutch origin. Only second-generation Ghanaian participants received more prescriptions compared to Dutch participants (aIRR 2.09, $95 \% \mathrm{Cl} 1.06$ to 4.12 ). Higher level of antibiotic knowledge was not significantly associated with the number of prescriptions (IRR $0.92,95 \% \mathrm{Cl} 0.85$ to 1.00 ).

Conclusions: Levels of antibiotic knowledge varied between ethnic groups, but a lower level of antibiotic knowledge did not correspond with a higher number of antibiotic prescriptions.

Keywords: Antibiotics, Antibiotic knowledge, Antibiotic use, Ethnic groups

\section{Background}

The emergence of antimicrobial resistance, along with the steady decline in antibiotic development, has been identified as a major health threat for the coming decade by the World Health Organization (WHO). Increase in antibiotic use is the main reason for this development [1] and as such, antibiotics should only be prescribed when there is a clear indication for use.

\footnotetext{
* Correspondence: evdulm@ggd.amsterdam.nl

${ }^{\dagger}$ Emelie C. Schuts and Eline van Dulm contributed equally to this work.

${ }^{2}$ Department of Infectious Diseases, Public Health Service Amsterdam,

Nieuwe Achtergracht 100, 1018, WT, Amsterdam, The Netherlands

Full list of author information is available at the end of the article
}

A recent meta-analysis showed a higher prevalence of antimicrobial resistance among migrants in Europe [2]. There is preliminary evidence in the Netherlands that the use of antibiotics also differs across ethnic groups, with a higher use of antibiotics among people of nonDutch origin [3]. The reason for this difference, however, is unclear. It could be explained by increased incidence of bacterial infections, but, to the best of our knowledge, there is no evidence to support this hypothesis. Alternatively, knowledge about antibiotic use might vary across ethnic groups. As expectations and knowledge of the patient could potentially drive a physician's decision to prescribe antibiotics, receiving prescriptions could also

(c) The Author(s). 2019 Open Access This article is distributed under the terms of the Creative Commons Attribution 4.0 International License (http://creativecommons.org/licenses/by/4.0/), which permits unrestricted use, distribution, and 
differ between ethnic groups [4-6]. There are also cultural-specific approaches to dealing with authority, being the physician in this setting, which have explained differences in antibiotic use between countries [7].

The HELIUS (Healthy life in an Urban Setting) study is a large-scale, population-based cohort study among different ethnic groups, which was established with the aim to investigate mechanisms underlying the impact of ethnicity on communicable and non-communicable diseases $[8,9]$. In 2018 , approximately $13 \%$ of the population of the Netherlands was of non-Western origin [10]. The largest non-Western population groups were individuals of Turkish (2.4\%), Moroccan (2.3\%) and Surinamese (2.0\%) descent [10]. In Amsterdam, approximately 36\% of the population in 2018 was of nonWestern descent [11]. The ethnic groups included in the HELIUS study are the largest ethnic minority groups of Amsterdam [9]. Amongst other data, data on antibiotic knowledge were collected. We were able to link these data at the individual level to data from a health insurance register on recent antibiotic use.

This study then provides a unique opportunity to determine whether knowledge about and use of antibiotics vary between ethnic groups, and if so, whether differences in antibiotic use can be attributed to differences in knowledge about antibiotics. We hypothesized that antibiotic use differs among ethnic groups as a result of differences in knowledge.

\section{Methods}

\section{Study population and design}

The HEalthy LIfe in an Urban Setting (HELIUS) study is a multiethnic cohort study conducted in Amsterdam, which focuses on cardiovascular disease (e.g. diabetes), mental health (e.g. depressive disorders), and infectious diseases $[8,9]$. In brief, baseline data collection took place in 2011-2015 and included people aged 18 to 70 years of Dutch, Surinamese, Ghanaian, Moroccan, and Turkish origin. A random sample of participants, stratified by ethnic origin, was taken from the municipality register of Amsterdam. Participants filled in an extensive self-administered questionnaire (variables included in the questionnaire are described elsewhere) [9] and underwent a physical examination during which biological samples were obtained [9]. No information was provided regarding appropriate antibiotic use. Between 2011 and 2015, 24,789 persons were included. Data collection procedures have been previously described in detail [9]. Both questionnaire data and physical examination data were available for 22 , 165 participants. The HELIUS study was conducted in accordance with the Declaration of Helsinki and was approved by the AMC Ethical Review Board. All participants provided written informed consent.
Ethnicity was defined according to the country of birth of the participant as well as that of their parents [12]. Specifically, a participant is considered to be of nonDutch ethnic origin if they fulfill either of the following criteria (i): they were born abroad and had at least one parent born abroad (first generation) or (ii) they were born in Netherlands but both their parents were born abroad (second generation). Dutch participants were born in the Netherlands and had both parents who were born in the Netherlands. After HELIUS data collection, the Surinamese group were further classified according to self-reported ethnic origin (obtained by questionnaire), into 'African Surinamese', 'SouthAsian Surinamese', 'Javanese Surinamese' and 'other/ unknown Surinamese'.

\section{Data linkage}

Permission to link participants' individual data to outside health registries was asked in the written informed consent form [8]. Of the 22,165 HELIUS participants, 19, 895 agreed. HELIUS data of these individuals were linked to reimbursement data from the Achmea insurance company (Achmea Health Database, AHD) from 2010 until 2015. The AHD, obtained from the largest health insurance company in Amsterdam, contains all healthcare expenditures of every insured participant, including medications. A trusted third party linked data on reimbursed antibiotic prescriptions using an encrypted social security number and returned data without any identifying information. Procedures were in accordance with the General Data Protection Regulation [13].

\section{Inclusion and exclusion criteria for present study}

Of the 22,165 participants, we excluded those of Javanese Surinamese or other/unknown Surinamese origin and those with another/unknown ethnic origin because of small participant numbers. For analyses on antibiotic use, we included those who gave permission for data linkage and could be linked to the AHD. To reduce bias for individuals with short-term insurance, we excluded those who were insured with Achmea for less than 365 days in the year preceding their HELIUS study visit.

\section{Outcome variables}

The primary outcomes were level of antibiotic knowledge and antibiotic use during the year prior to the HELIUS visit. Level of antibiotic knowledge was based on five questions, used in other studies $[4,6,14]$, which asked the perceived necessity (yes/no) for antibiotic treatment during influenza-like illness, pneumonia, fever, sore throat and bronchitis. Using these questions, we created an overall knowledge score of antibiotic use by summing the total number of correct responses, 
resulting in a score ranging from 0 to 5 . A twoparameter logistic regression model was fitted to the five binary items based on the assumptions of item response theory (see Additional file 1). From this model, "higher" and "lower" levels of antibiotic knowledge were defined by a knowledge score of $\geq 4$ and $<4$, respectively.

Antibiotic use was obtained from linked AHD data and was based on the total number of reimbursed antibiotics (classified by ATC code J01; anti-infectives for systemic use) dispensed by community pharmacies from 2010 until 2015. We evaluated antibiotic use (yes/no) in the year prior to the HELIUS study visit, as well as the number of antibiotic prescriptions over the past year and during the entire insured period.

\section{Other variables}

Independent variables were obtained from the HELIUS study questionnaire (migration generation; sex; age; level of education; marital status; self-reported medical conditions; smoking; alcohol consumption; difficulty with the Dutch language and perceived health) and physical examination (body mass index $\left(B M I, \mathrm{~kg} / \mathrm{m}^{2}\right)$ ). Variables on antibiotic-related behavior were: not having finished antibiotic treatment; having saved antibiotics for later; and ever having asked the general practitioner (GP) for antibiotics. Definitions and grouping of variables are extensively described elsewhere [8].

\section{Statistical analyses}

Sociodemographics, health status, antibiotic knowledge level and questions on antibiotic use were presented by ethnicity. To assess selection bias resulting from AHD data linkage, the same variables were compared between participants who were successfully versus unsuccessfully linked. Comparisons between ethnic groups were made using a Pearson's $\chi^{2}$ or Fisher exact test for categorical data and Kruskal-Wallis rank test for continuous variables.

Analysis on level of antibiotic knowledge included all HELIUS participants with available data. Odds ratios (OR) comparing levels of antibiotic knowledge across determinants and their 95\% confidence intervals (CI) were estimated using logistic regression. All variables with an associated $p$-value $<0.2$ in univariable analyses were included in a full multivariable model and variables with a $p$-value above this level were removed in backwards-stepwise fashion. Given that the research aim was to determine differences between ethnicity, ethnic groups were forced in all models. This multivariable approach was chosen to not only assess other variables associated with antibiotic knowledge, but also to understand the extent of confounding bias when assessing the relationship between ethnicity and outcome variables.
Analysis on antibiotic use in the year prior to HELIUS study visit included all HELIUS participants who were linked to the AHD and were insured for at least 365 days with Achmea in the year prior to their HELIUS study visit. Determinants for having received $\geq 1$ antibiotic prescription were assessed using logistic regression. The same multivariable approach as above was used for this outcome. We also compared antibiotic use during the entire period insured at Achmea versus the year prior to HELIUS study visit to assess differences when considering longer time periods.

Determinants for the total number of antibiotic prescriptions were then evaluated. As this outcome contained a high proportion of zero values and was overdispersed, we used a zero-inflated negative binomial regression model. This model contains two parts: one accounting for zero values in the count distribution (zeroinflated) and another accounting for the over-dispersed count distribution (negative binomial). Covariates for the zero-inflated part were determined a priori from the risk-factor analysis on $\geq 1$ antibiotic prescription. Covariates for the negative binomial part were selected from covariates with a $p$-value $<0.2$ in univariable analyses and variables above this $p$-value were removed in backwards-stepwise fashion. Incidence risk ratios (IRR) comparing the number of antibiotics prescribed over the past year across levels of determinants were estimated from this model.

Multicollinearity was verified using variance inflation factors, while any variable with an inflation factor of $\geq 4$ was considered multicollinear and excluded from the model. To understand whether the association between ethnicity and outcome was modified by demographic variables, interaction between ethnicity and other demographic variables was also assessed in all multivariable models.

The three variables involving antibiotic-related behavior were not initially considered in the final multivariable models. To assess whether ethnic differences in antibiotic use could be explained by patterns of antibioticrelated behavior, additional multivariable models including these variables were constructed for the endpoints (i) having received $\geq 1$ antibiotic prescription and (ii) total number of antibiotic prescriptions.

Figure 1 provides an overview of all descriptive analysis and modeling used in the study. Significance was determined using a $p$-value $<0.05$. All analyses were conducted with Stata 13.1 (StataCorp., College Station, Texas, USA).

\section{Results}

Participants

Of the 22,165 HELIUS participants with available data, 21,617 were eligible after applying exclusion criteria. 


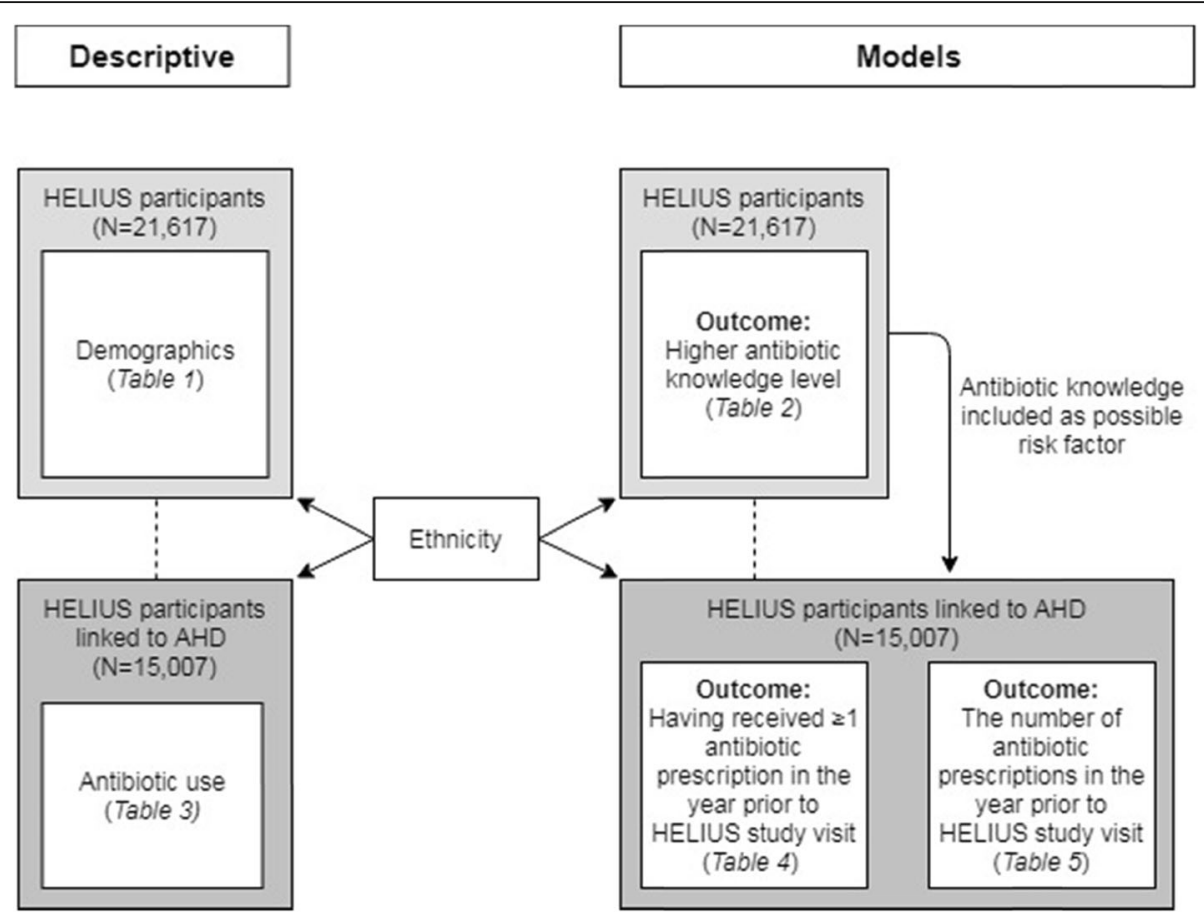

Fig. 1 Overview of descriptive analysis and models used in the study. Abbreviations: HELIUS - Healthy Life in an Urban Setting; AHD - Achmea Health Database

Their baseline characteristics, stratified by ethnicity, are shown in Table 1. Median age of participants was 46 years (IQR 34 to 55 ) and 58\% were women. The proportion of several medical conditions predisposing individuals to antibiotic treatment differed by ethnicity. Of these conditions, South-Asian Surinamese participants had the highest prevalence of self-reported diabetes mellitus (17\%) and cerebrovascular accident (CVA) (6\%) over the last 12 months. Turkish individuals had more prevalent artery stenosis (10\%), severe or chronic fatigue (45\%) and respiratory diseases (15\%), whereas Ghanaians more frequently reported high blood pressure (33\%). Excellent perceived health was reported in 12\% of Dutch participants in contrast to $3.3 \%$ of Turkish participants.

\section{Ethnic differences in antibiotic knowledge}

In several ethnic groups, there were substantial proportions of individuals reporting the need to be treated with antibiotics for illnesses without indication, as shown in Table 1. The number of people reporting to have been treated with antibiotics and not having regularly completed their antibiotic treatment was low across all ethnic groups, ranging from $0.1 \%$ in Dutch participants to $2.1 \%$ in Ghanaian participants. Few individuals regularly saved their antibiotics for later use, ranging from $<0.1 \%$ in Dutch participants to $0.3 \%$ in Turkish participants. The percentage of participants having regularly asked their GP for antibiotics ranged from $0.6 \%$ in African Surinamese participants to $1.9 \%$ in Turkish and Moroccan participants.

As shown in Table 2, there was a significantly lower odds of individuals with higher level of antibiotic knowledge among all non-Dutch ethnic groups compared to Dutch individuals (overall $p<0.001$ ) (Table 2). Across all non-Dutch groups, second-generation participants had a higher level of antibiotic knowledge than first-generation participants; however, results remained significantly lower compared to the Dutch group.

In multivariable analysis, all ethnic minority groups had lower odds for higher level of antibiotic knowledge compared to Dutch (overall $p<0.001$ ), although the effect for second-generation Ghanaian participants was not statistically significant. The odds for higher level of antibiotic knowledge were higher in all age groups $>25$ years of age (except for those 265 ) when compared to $\leq 25$ years of age. Furthermore, women had a significantly higher odds of having a higher level of antibiotic knowledge compared to males. Lower odds for a higher level of antibiotic knowledge were found for the following medical conditions: myocardial Infarction (MI), severe or chronic fatigue, respiratory diseases and having a BMI $\geq 25$. Lower odds for higher level of antibiotic knowledge were also seen among individuals who regularly or occasionally requested antibiotics from their GP or who regularly or occasionally did not finish treatment. 
Table 1 Characteristics of the HELIUS study population $(N=21,617)$ by ethnicity

\begin{tabular}{|c|c|c|c|c|c|c|c|c|c|c|c|c|}
\hline \multirow{3}{*}{$\begin{array}{l}\text { Variables }^{\mathrm{a}} \\
\text { Sociodemographics }\end{array}$} & \multicolumn{12}{|c|}{ Ethnicity } \\
\hline & \multicolumn{2}{|c|}{$\begin{array}{l}\text { Dutch } \\
(N=4564)\end{array}$} & \multicolumn{2}{|c|}{$\begin{array}{l}\text { South-Asian } \\
\text { Surinamese } \\
(N=3043)\end{array}$} & \multicolumn{2}{|c|}{$\begin{array}{l}\text { African } \\
\text { Surinamese } \\
(N=4151)\end{array}$} & \multicolumn{2}{|c|}{$\begin{array}{l}\text { Ghanaian } \\
(N=2339)\end{array}$} & \multicolumn{2}{|c|}{$\begin{array}{l}\text { Turkish } \\
(N=3614)\end{array}$} & \multicolumn{2}{|c|}{$\begin{array}{l}\text { Moroccan } \\
(N=3906)\end{array}$} \\
\hline & & & & & & & & & & & & \\
\hline Female sex & 2475 & $54 \%$ & 1672 & $55 \%$ & 2535 & $61 \%$ & 1434 & $61 \%$ & 1980 & $55 \%$ & 2392 & $61 \%$ \\
\hline Age in years, median (IQR) & 47 & $(34-58)$ & 48 & $(35-56)$ & 50 & $(40-57)$ & 47 & $(38-53)$ & 42 & $(31-50)$ & 40 & $(30-50)$ \\
\hline \multicolumn{13}{|l|}{ Migration generation } \\
\hline 1st generation & N.A. & N.A. & 2328 & $77 \%$ & 3468 & $84 \%$ & 2231 & $95 \%$ & 2544 & $70 \%$ & 2680 & $69 \%$ \\
\hline 2nd generation & N.A. & N.A. & 715 & $23 \%$ & 683 & $16 \%$ & 108 & $4.6 \%$ & 1080 & $30 \%$ & 1226 & $31 \%$ \\
\hline \multicolumn{13}{|l|}{ Educational level } \\
\hline Unknown & 25 & $0.6 \%$ & 16 & $0.5 \%$ & 36 & $0.9 \%$ & 42 & $1.8 \%$ & 38 & $1.1 \%$ & 38 & $1.0 \%$ \\
\hline No school/elementary school & 150 & $3.3 \%$ & 437 & $14 \%$ & 231 & $6 \%$ & 660 & $28 \%$ & 1135 & $31 \%$ & 1205 & $31 \%$ \\
\hline $\begin{array}{l}\text { Lower vocational/lower secondary } \\
\text { school }\end{array}$ & 646 & $14 \%$ & 1010 & $33 \%$ & 1477 & $36 \%$ & 917 & $39 \%$ & 889 & $25 \%$ & 694 & $18 \%$ \\
\hline $\begin{array}{l}\text { Intermediate vocational/ intermediate } \\
\text { secondary school }\end{array}$ & 994 & $22 \%$ & 885 & $29 \%$ & 1464 & $35 \%$ & 578 & $25 \%$ & 1020 & $28 \%$ & 1294 & $33 \%$ \\
\hline Higher vocational/university & 2749 & $60 \%$ & 695 & $23 \%$ & 943 & $23 \%$ & 142 & $6 \%$ & 532 & $15 \%$ & 675 & $17 \%$ \\
\hline \multicolumn{13}{|l|}{ Marital status } \\
\hline Married/registered partnership & 1724 & $38 \%$ & 1043 & $34 \%$ & 766 & $19 \%$ & 420 & $18 \%$ & 2208 & $61 \%$ & 2285 & $59 \%$ \\
\hline Cohabiting & 914 & $20 \%$ & 311 & $10 \%$ & 441 & $11 \%$ & 427 & $19 \%$ & 132 & $3.7 \%$ & 110 & $2.8 \%$ \\
\hline Unmarried/never married & 1474 & $32 \%$ & 1001 & $33 \%$ & 2231 & $54 \%$ & 779 & $34 \%$ & 761 & $21 \%$ & 1010 & $26 \%$ \\
\hline Divorced/separated & 356 & $8 \%$ & 580 & $19 \%$ & 617 & $15 \%$ & 656 & $28 \%$ & 407 & $11 \%$ & 414 & $11 \%$ \\
\hline Widow/widower & 87 & $1.9 \%$ & 92 & $3.0 \%$ & 65 & $1.6 \%$ & 23 & $1.0 \%$ & 90 & $2.5 \%$ & 69 & $1.8 \%$ \\
\hline \multicolumn{13}{|l|}{ Health status } \\
\hline \multicolumn{13}{|c|}{ Self-reported medical conditions (previous 12 months) } \\
\hline Diabetes mellitus & 102 & $2.2 \%$ & 521 & $17 \%$ & 419 & $10 \%$ & 185 & $8 \%$ & 336 & $9 \%$ & 389 & $10 \%$ \\
\hline $\begin{array}{l}\text { CVA/one-sided loss of bodily } \\
\text { function } \leq 1 \text { day }\end{array}$ & 160 & $3.5 \%$ & 212 & $7 \%$ & 261 & $6 \%$ & 95 & $4.1 \%$ & 196 & $5 \%$ & 195 & $5 \%$ \\
\hline $\begin{array}{l}\text { Ml incl. Zhalf hour chest pain or } \\
\text { dotter/bypass operation }\end{array}$ & 233 & $5 \%$ & 491 & $16 \%$ & 440 & $11 \%$ & 225 & $10 \%$ & 591 & $16 \%$ & 476 & $12 \%$ \\
\hline Severe heart condition & 67 & $1.5 \%$ & 120 & $4.0 \%$ & 105 & $2.5 \%$ & 75 & $3.2 \%$ & 153 & $4.3 \%$ & 58 & $1.5 \%$ \\
\hline Malignant disorder & 103 & $2.3 \%$ & 70 & $2.3 \%$ & 85 & $2.1 \%$ & 33 & $1.4 \%$ & 73 & $2.0 \%$ & 46 & $1.2 \%$ \\
\hline Severe or chronic fatigue & 633 & $14 \%$ & 1032 & $34 \%$ & 956 & $23 \%$ & 186 & $8 \%$ & 1602 & $45 \%$ & 1465 & $38 \%$ \\
\hline High blood pressure & 534 & $12 \%$ & 720 & $24 \%$ & 1230 & $30 \%$ & 770 & $33 \%$ & 610 & $17 \%$ & 546 & $14 \%$ \\
\hline Artery stenosis & 85 & $1.9 \%$ & 193 & $6 \%$ & 181 & $4.4 \%$ & 115 & $5 \%$ & 348 & $10 \%$ & 200 & $5 \%$ \\
\hline Respiratory diseases & 345 & $8 \%$ & 433 & $14 \%$ & 354 & $9 \%$ & 117 & $5 \%$ & 556 & $15 \%$ & 446 & $11 \%$ \\
\hline Serious/persistent intestinal disorders & 249 & $5 \%$ & 248 & $8 \%$ & 308 & $7 \%$ & 70 & $3.0 \%$ & 433 & $12 \%$ & 391 & $10 \%$ \\
\hline Psoriasis & 136 & $3.0 \%$ & 168 & $6 \%$ & 128 & $3.1 \%$ & 71 & $3.1 \%$ & 154 & $4.3 \%$ & 121 & $3.1 \%$ \\
\hline (Chronic) eczema & 420 & $9 \%$ & 406 & $13 \%$ & 370 & $9 \%$ & 71 & $3.1 \%$ & 471 & $13 \%$ & 423 & $11 \%$ \\
\hline Incontinence & 309 & $7 \%$ & 326 & $11 \%$ & 342 & $8 \%$ & 108 & $4.7 \%$ & 464 & $13 \%$ & 300 & $8 \%$ \\
\hline Body Mass Index $\left(\mathrm{kg} / \mathrm{m}^{2}\right)$, median (IQR) & 24.1 & $\begin{array}{l}(21.9- \\
26.7)\end{array}$ & 25.7 & $\begin{array}{l}(23.2- \\
28.8)\end{array}$ & 27.0 & $\begin{array}{l}(23.9- \\
30.8)\end{array}$ & 27.9 & $\begin{array}{l}(25.0- \\
31.2)\end{array}$ & 27.9 & $\begin{array}{l}(24.6- \\
31.7)\end{array}$ & 27.0 & $\begin{array}{l}(23.9- \\
30.7)\end{array}$ \\
\hline \multicolumn{13}{|l|}{ Smoking } \\
\hline Yes & 1129 & $25 \%$ & 861 & $28 \%$ & 1309 & $32 \%$ & 104 & $4.5 \%$ & 1240 & $35 \%$ & 525 & $13 \%$ \\
\hline No, never & 1689 & $37 \%$ & 1758 & $58 \%$ & 2016 & $49 \%$ & 2027 & $87 \%$ & 1700 & $47 \%$ & 2874 & $74 \%$ \\
\hline No, but ever & 1737 & $38 \%$ & 413 & $14 \%$ & 805 & $19 \%$ & 191 & $8 \%$ & 648 & $18 \%$ & 492 & $13 \%$ \\
\hline \multicolumn{13}{|l|}{ Alcohol consumption } \\
\hline Never & 297 & $7 \%$ & 1072 & $35 \%$ & 1002 & $24 \%$ & 806 & $35 \%$ & 2414 & $67 \%$ & 3265 & $84 \%$ \\
\hline
\end{tabular}


Table 1 Characteristics of the HELIUS study population $(N=21,617)$ by ethnicity (Continued)

\begin{tabular}{|c|c|c|c|c|c|c|c|c|c|c|c|c|}
\hline \multirow{3}{*}{$\begin{array}{l}\text { Variables }^{\mathrm{a}} \\
\text { Not in previous } 12 \text { months }\end{array}$} & \multicolumn{12}{|c|}{ Ethnicity } \\
\hline & \multicolumn{2}{|c|}{$\begin{array}{l}\text { Dutch } \\
(N=4564)\end{array}$} & \multicolumn{2}{|c|}{$\begin{array}{l}\text { South-Asian } \\
\text { Surinamese } \\
(N=3043)\end{array}$} & \multicolumn{2}{|c|}{$\begin{array}{l}\text { African } \\
\text { Surinamese } \\
(N=4151)\end{array}$} & \multicolumn{2}{|c|}{$\begin{array}{l}\text { Ghanaian } \\
(N=2339)\end{array}$} & \multicolumn{2}{|c|}{$\begin{array}{l}\text { Turkish } \\
(N=3614)\end{array}$} & \multicolumn{2}{|c|}{$\begin{array}{l}\text { Moroccan } \\
(N=3906)\end{array}$} \\
\hline & 110 & $2.4 \%$ & 251 & $8 \%$ & 292 & $7 \%$ & 408 & $18 \%$ & 358 & $10 \%$ & 338 & $9 \%$ \\
\hline Monthly or less & 436 & $10 \%$ & 758 & $25 \%$ & 1242 & $30 \%$ & 508 & $22 \%$ & 367 & $10 \%$ & 127 & $3.3 \%$ \\
\hline 2-4 times per month & 894 & $20 \%$ & 541 & $18 \%$ & 873 & $21 \%$ & 291 & $13 \%$ & 257 & $7 \%$ & 87 & $2.2 \%$ \\
\hline 2-3 times per week & 1413 & $31 \%$ & 262 & $9 \%$ & 439 & $11 \%$ & 193 & $8 \%$ & 132 & $3.7 \%$ & 56 & $1.4 \%$ \\
\hline$\geq 4$ times per week & 1408 & $31 \%$ & 147 & $5 \%$ & 272 & $7 \%$ & 109 & $4.7 \%$ & 57 & $1.6 \%$ & 16 & $0.4 \%$ \\
\hline \multicolumn{13}{|l|}{ Difficulty with Dutch language } \\
\hline Yes & N.A. & N.A. & 711 & $23 \%$ & 520 & $13 \%$ & 1926 & $83 \%$ & 2136 & $60 \%$ & 1774 & $46 \%$ \\
\hline \multicolumn{13}{|l|}{ Perceived health } \\
\hline Excellent & 541 & $12 \%$ & 162 & $5 \%$ & 303 & $7 \%$ & 226 & $10 \%$ & 117 & $3.3 \%$ & 166 & $4.3 \%$ \\
\hline Very good & 1381 & $30 \%$ & 310 & $10 \%$ & 571 & $14 \%$ & 458 & $20 \%$ & 383 & $11 \%$ & 384 & $10 \%$ \\
\hline Good & 2205 & $48 \%$ & 1623 & $53 \%$ & 2335 & $56 \%$ & 1180 & $51 \%$ & 1871 & $52 \%$ & 1871 & $48 \%$ \\
\hline Mediocre & 402 & $9 \%$ & 811 & $27 \%$ & 834 & $20 \%$ & 383 & $16 \%$ & 921 & $26 \%$ & 1223 & $31 \%$ \\
\hline Bad & 28 & $0.6 \%$ & 131 & $4.3 \%$ & 101 & $2.4 \%$ & 88 & $3.8 \%$ & 307 & $9 \%$ & 241 & $6 \%$ \\
\hline \multicolumn{13}{|l|}{ Antibiotics } \\
\hline \multicolumn{13}{|l|}{ Knowledge concerning antibiotics ${ }^{b}$} \\
\hline Antibiotics effective for influenza & 324 & $7 \%$ & 554 & $19 \%$ & 592 & $15 \%$ & 658 & $29 \%$ & 744 & $21 \%$ & 648 & $18 \%$ \\
\hline Antibiotics effective for pneumonia & 4166 & $92 \%$ & 2304 & $77 \%$ & 3114 & $77 \%$ & 1312 & $58 \%$ & 2587 & $73 \%$ & 2741 & $73 \%$ \\
\hline Antibiotics effective for fever ${ }^{c}$ & 689 & $15 \%$ & 552 & $19 \%$ & 679 & $17 \%$ & 586 & $26 \%$ & 898 & $26 \%$ & 614 & $17 \%$ \\
\hline Antibiotics effective for sore throat ${ }^{c}$ & 672 & $15 \%$ & 760 & $26 \%$ & 1089 & $27 \%$ & 720 & $32 \%$ & 1203 & $34 \%$ & 978 & $26 \%$ \\
\hline Antibiotics effective for bronchitis & 2246 & $50 \%$ & 1385 & $50 \%$ & 1862 & $46 \%$ & 919 & $41 \%$ & 1235 & $35 \%$ & 1485 & $40 \%$ \\
\hline Higher level of antibiotic knowledge ${ }^{d}$ & 3638 & $80 \%$ & 1996 & $68 \%$ & 2737 & $69 \%$ & 1248 & $57 \%$ & 2128 & $62 \%$ & 2528 & $70 \%$ \\
\hline \multicolumn{13}{|l|}{ Did not finish antibiotic treatment } \\
\hline Yes, regularly & 5 & $0.1 \%$ & 49 & $1.6 \%$ & 44 & $1.1 \%$ & 48 & $2.1 \%$ & 41 & $1.2 \%$ & 44 & $1.1 \%$ \\
\hline Yes, occasionally & 332 & $7 \%$ & 312 & $10 \%$ & 527 & $13 \%$ & 174 & $8 \%$ & 424 & $12 \%$ & 445 & $12 \%$ \\
\hline Always finished or no antibiotics & 4203 & $93 \%$ & 2646 & $88 \%$ & 3524 & $86 \%$ & 2053 & $90 \%$ & 3104 & $87 \%$ & 3361 & $87 \%$ \\
\hline \multicolumn{13}{|l|}{ Saved antibiotics for later } \\
\hline Yes, regularly & 2 & $0.0 \%$ & 7 & $0.2 \%$ & 9 & $0.2 \%$ & 5 & $0.2 \%$ & 10 & $0.3 \%$ & 6 & $0.2 \%$ \\
\hline Yes, occasionally & 37 & $0.8 \%$ & 45 & $1.5 \%$ & 68 & $1.7 \%$ & 62 & $2.7 \%$ & 60 & $1.7 \%$ & 46 & $1.2 \%$ \\
\hline No, never & 297 & $7 \%$ & 304 & $10 \%$ & 492 & $12 \%$ & 146 & $6 \%$ & 387 & $11 \%$ & 430 & $11 \%$ \\
\hline Not applicable (no antibiotics) & 4203 & $93 \%$ & 2646 & $88 \%$ & 3524 & $86 \%$ & 2053 & $91 \%$ & 3104 & $87 \%$ & 3361 & $87 \%$ \\
\hline \multicolumn{13}{|l|}{ Ever asked GP for antibiotics } \\
\hline Yes, regularly & 38 & $0.8 \%$ & 34 & $1.1 \%$ & 26 & $0.6 \%$ & 36 & $1.6 \%$ & 67 & $1.9 \%$ & 71 & $1.9 \%$ \\
\hline Yes, occasionally & 824 & $18 \%$ & 491 & $16 \%$ & 607 & $15 \%$ & 401 & $18 \%$ & 734 & $21 \%$ & 634 & $17 \%$ \\
\hline No, never & 3674 & $81 \%$ & 2482 & $83 \%$ & 3441 & $84 \%$ & 1835 & $81 \%$ & 2744 & $77 \%$ & 3074 & $81 \%$ \\
\hline
\end{tabular}

Missing data, $n$ : marital status 128; diabetes 78; stroke 55; myocardial infarction 33; heart condition 83; malignant disorders 137; fatigue 145; high blood pressure 101; artery stenosis 140; respiratory diseases 115; bowel diseases 115; psoriasis 98; eczema 117; incontinence 126; BMI 23; smoking 107; alcohol 127; perceived health 61; $A B$ effective for influenza 614; $A B$ effective for pneumonia $477 ; A B$ effective for fever $685 ; A B$ effective for sore throat 625; $A B$ effective for bronchitis 663; asked GP for $A B$ 414; did not finish treatment 292; saved $A B 325$

Abbreviations: IQR Inter Quartile Range, CVA Cerebro Vascular Accident, MI Myocardial infarction, N.A. Not Applicable, GP General Practitioner N.A. Not applicable (categories not applicable due to Dutch ethnicity)

${ }^{a}$ All variables are reported as $n(\%)$, unless otherwise indicated

"Answered "yes" to the statements below

'The Dutch General Practitioners guidelines (and those of other European countries) advise against the use of antibiotics for fever in general or sore throat, as they usually constitute viral infections, with only a few exceptions in both cases. Therefore, antibiotics are in general not appropriate for these conditions

${ }^{d}$ Based on a summed score with cutoff determined by an Item Response Theory model ( $\geq 4$ out of 5 antibiotic knowledge questions correctly answered was considered as having a higher level of knowledge) 
Table 2 Variables associated with higher antibiotic knowledge in HELIUS study population $(N=21,617)$ (logistic regression analysis)

\begin{tabular}{|c|c|c|c|c|c|c|}
\hline & \multicolumn{3}{|c|}{ Univariable } & \multicolumn{3}{|c|}{ Multivariable $\left(N=20,081^{a}\right) \#$} \\
\hline & $\mathrm{OR}$ & $(95 \% \mathrm{Cl})$ & $P$-values & $\mathrm{aOR}$ & $(95 \% \mathrm{Cl})$ & $P$-values \\
\hline \multicolumn{7}{|l|}{ Sociodemographics } \\
\hline Ethnicity & & & $<.001$ & & & $<.001$ \\
\hline Dutch & Ref & & & Ref & & \\
\hline \multicolumn{7}{|l|}{ South-Asian Surinamese } \\
\hline 1st generation & 0.49 & $0.44-0.55$ & & 0.53 & $0.47-0.60$ & \\
\hline 2nd generation & 0.56 & $0.47-0.67$ & & 0.60 & $0.50-0.73$ & \\
\hline \multicolumn{7}{|l|}{ African Surinamese } \\
\hline 1st generation & 0.51 & $0.46-0.57$ & & 0.53 & $0.47-0.59$ & \\
\hline 2nd generation & 0.75 & $0.62-0.91$ & & 0.79 & $0.64-0.96$ & \\
\hline \multicolumn{7}{|l|}{ Ghanaian } \\
\hline 1st generation & 0.31 & $0.27-0.34$ & & 0.31 & $0.27-0.35$ & \\
\hline 2nd generation & 0.64 & $0.41-0.98$ & & 0.74 & $0.47-1.18$ & \\
\hline \multicolumn{7}{|l|}{ Turkish } \\
\hline 1st generation & 0.35 & $0.31-0.39$ & & 0.40 & $0.36-0.45$ & \\
\hline 2nd generation & 0.56 & $0.48-0.65$ & & 0.62 & $0.53-0.74$ & \\
\hline \multicolumn{7}{|l|}{ Moroccan } \\
\hline 1st generation & 0.51 & $0.45-0.57$ & & 0.56 & $0.50-0.63$ & \\
\hline 2nd generation & 0.71 & $0.61-0.83$ & & 0.75 & $0.63-0.89$ & \\
\hline Female sex & 1.18 & $1.11-1.25$ & $<.001$ & 1.32 & $1.23-1.40$ & $<.001$ \\
\hline Age & & & $<.001$ & & & $<.001$ \\
\hline$<25$ years & Ref & & & Ref & & \\
\hline $25-34$ years & 1.24 & $1.01-1.34$ & & 1.32 & $1.16-1.50$ & \\
\hline $35-44$ years & 0.99 & $0.81-1.05$ & & 1.30 & $1.14-1.49$ & \\
\hline $45-54$ years & 0.85 & $0.71-0.91$ & & 1.19 & $1.04-1.37$ & \\
\hline $55-64$ years & 0.95 & $0.78-1.02$ & & 1.26 & $1.08-1.45$ & \\
\hline$\geq 65$ years & 1.06 & $0.84-1.22$ & & 1.15 & $0.95-1.39$ & \\
\hline Educational level & & & $<.001$ & & & \\
\hline Unknown & Ref & & & & & \\
\hline No school/elementary school & 1.10 & $0.79-1.55$ & & & & \\
\hline Lower vocational/lower secondary school & 1.27 & $0.91-1.77$ & & & & \\
\hline Intermediate vocational/ intermediate secondary school & 1.54 & $1.11-2.15$ & & & & \\
\hline Higher vocational/university & 2.06 & $1.47-2.87$ & & & & \\
\hline Marital status & & & $<.001$ & & & \\
\hline Married/registered partnership & Ref & & & & & \\
\hline Cohabiting & 1.15 & $1.04-1.27$ & & & & \\
\hline Unmarried/never married & 1.08 & $1.01-1.16$ & & & & \\
\hline Divorced/separated & 0.81 & $0.74-0.89$ & & & & \\
\hline Widow/widower & 1.10 & $0.88-1.36$ & & & & \\
\hline \multicolumn{7}{|l|}{ Health status } \\
\hline \multicolumn{7}{|l|}{ Self-reported medical conditions (previous 12 months) } \\
\hline Diabetes mellitus & 0.70 & $0.63-0.77$ & $<.001$ & & & \\
\hline CVA/one-sided loss of bodily function $\leq 1$ day & 0.85 & $0.75-0.97$ & .015 & & & \\
\hline Ml incl. Zhalf hour chest pain or dotter/bypass operation & 0.71 & $0.65-0.77$ & $<.001$ & 0.89 & $0.81-0.98$ & .017 \\
\hline
\end{tabular}


Table 2 Variables associated with higher antibiotic knowledge in HELIUS study population $(N=21,617)$ (logistic regression analysis) (Continued)

\begin{tabular}{|c|c|c|c|c|c|c|}
\hline & \multicolumn{3}{|c|}{ Univariable } & \multicolumn{3}{|c|}{ Multivariable $\left(N=20,081^{\mathrm{a}}\right) \#$} \\
\hline & $\mathrm{OR}$ & $(95 \% \mathrm{Cl})$ & $P$-values & $\mathrm{aOR}$ & $(95 \% \mathrm{Cl})$ & $P$-values \\
\hline Severe heart condition & 0.61 & $0.52-0.73$ & $<.001$ & & & \\
\hline Malignant disorder & 0.97 & $0.78-1.20$ & .752 & & & \\
\hline Severe or chronic fatigue & 0.80 & $0.75-0.85$ & $<.001$ & 0.89 & $0.82-0.95$ & .001 \\
\hline High blood pressure & 0.78 & $0.73-0.84$ & $<.001$ & & & \\
\hline Artery stenosis & 0.69 & $0.61-0.78$ & $<.001$ & & & \\
\hline Respiratory diseases & 0.68 & $0.62-0.75$ & $<.001$ & 0.80 & $0.72-0.88$ & $<.001$ \\
\hline Serious/persistent intestinal disorders & 0.88 & $0.79-0.98$ & .024 & & & \\
\hline Psoriasis & 0.76 & $0.66-0.89$ & .001 & & & \\
\hline (Chronic) eczema & 0.93 & $0.84-1.02$ & .121 & & & \\
\hline Incontinence & 0.84 & $0.76-0.93$ & .001 & & & \\
\hline Body Mass Index & & & $<.001$ & & & .001 \\
\hline$<18.5$ & Ref & & & Ref & & \\
\hline $18.5-25$ & 1.03 & $0.81-1.31$ & & 1.02 & $0.80-1.31$ & \\
\hline $25-30$ & 0.80 & $0.63-1.01$ & & 0.96 & $0.75-1.24$ & \\
\hline $30-40$ & 0.67 & $0.52-0.85$ & & 0.86 & $0.66-1.11$ & \\
\hline$\geq 40$ & 0.58 & $0.43-0.78$ & & 0.78 & $0.57-1.08$ & \\
\hline Smoking & & & $<.001$ & & & \\
\hline Yes & Ref & & & & & \\
\hline No, never & 1.03 & $0.96-1.11$ & & & & \\
\hline No, but ever & 1.19 & $1.09-1.30$ & & & & \\
\hline Alcohol usage & & & $<.001$ & & & \\
\hline Never & Ref & & & & & \\
\hline Not in previous 12 months & 0.89 & $0.80-1.00$ & & & & \\
\hline Monthly or less & 1.10 & $1.01-1.20$ & & & & \\
\hline 2-4 times per month & 1.27 & $1.16-1.40$ & & & & \\
\hline 2-3 times per week & 1.35 & $1.22-1.49$ & & & & \\
\hline$\geq 4$ times per week & 1.59 & $1.42-1.78$ & & & & \\
\hline Difficulty with Dutch language & & & $<.001$ & & & \\
\hline No & Ref & & & & & \\
\hline Yes & 0.65 & $0.61-0.69$ & & & & \\
\hline Not applicable & 1.70 & $1.56-1.85$ & & & & \\
\hline Perceived health & & & $<.001$ & & & \\
\hline Excellent & Ref & & & & & \\
\hline Very good & 0.97 & $0.85-1.12$ & & & & \\
\hline Good & 0.83 & $0.73-0.93$ & & & & \\
\hline Mediocre & 0.63 & $0.56-0.72$ & & & & \\
\hline Bad & 0.48 & $0.40-0.57$ & & & & \\
\hline \multicolumn{7}{|l|}{ Antibiotics } \\
\hline Ever asked GP for antibiotics & & & $<.001$ & & & $<.001$ \\
\hline No, never & Ref & & & Ref & & \\
\hline Yes, regularly & 0.51 & $0.40-0.65$ & & 0.60 & $0.46-0.77$ & \\
\hline Yes, occasionally & 0.57 & $0.53-0.61$ & & 0.59 & $0.55-0.64$ & \\
\hline
\end{tabular}


Table 2 Variables associated with higher antibiotic knowledge in HELIUS study population $(N=21,617)$ (logistic regression analysis) (Continued)

\begin{tabular}{|c|c|c|c|c|c|c|}
\hline & \multicolumn{3}{|c|}{ Univariable } & \multicolumn{3}{|c|}{ Multivariable $\left(N=20,081^{\mathrm{a}}\right) \#$} \\
\hline & OR & $(95 \% \mathrm{Cl})$ & $P$-values & $\mathrm{aOR}$ & $(95 \% \mathrm{Cl})$ & $P$-values \\
\hline Did not finish treatment & & & $<.001$ & & & $<.001$ \\
\hline Always finished or no antibiotics & Ref & & & Ref & & \\
\hline Yes, regularly & 0.51 & $0.39-0.67$ & & 0.71 & $0.54-0.94$ & \\
\hline Yes, occasionally & 0.73 & $0.66-0.80$ & & 0.80 & $0.73-0.88$ & \\
\hline
\end{tabular}

Abbreviations: OR Odds Ratio, aOR adjusted Odds Ratio, CI Confidence Interval, CVA Cerebro Vascular Accident, MI Myocardial infarction, GP General Practitioner ${ }^{a}$ Fewer observations in the multivariable model than in the total study population were due to missing observations on certain covariates \#We found significant interactions between ethnicity and sex $(p=0.007)$ and ethnicity and age $(p=0.047)$

\section{Ethnic difference in antibiotic use}

Of the 19,895 HELIUS participants consenting to link their data to other health registries, 15,461 were linked to the AHD (77.7\%). Of these 15,461 participants, 15,007 (97\%) were insured for $\geq 365$ days in the year prior to their HELIUS study visit. Additional file 2: Table S1 shows the characteristics of the study participants linked versus not linked to the AHD. Participants present in the AHD register had a lower level of education, higher prevalence of medical conditions, and less often had higher levels of antibiotic knowledge.

Table 3 describes antibiotic use according to ethnicity for participants registered in the AHD. In total, 31,530 antibiotic prescriptions were recorded over the study period. The proportion of participants receiving $\geq 1$ antibiotic prescription in the year prior to their HELIUS study visit was highest among first-generation Turkish participants (25\%) and was comparably high among second-generation Ghanaian and first-generation Moroccan participants (both 25\%). The proportion of participants receiving $\geq 1$ antibiotic prescription in the year prior to the HELIUS study visit was lowest in Dutch and second generation South-Asian Surinamese participants (both 16\%).

When considering the entire period during which participants were insured at Achmea prior to the HELIUS study visit (median 6.0 years, IQR 5.0 to 6.0), the proportion of participants receiving $\geq 1$ antibiotic prescription was highest among first generation Turkish participants (69\%) and lowest in second-generation Ghanaian participants (49\%). The mean number of prescriptions during the entire insured period was comparable to the mean number of prescriptions in the year prior to HELIUS study visit for all ethnic groups (Table 2).

\section{Determinants of antibiotic use and number of prescriptions}

Table 4 shows the results from the analysis on the association between ethnicity and having received $\geq 1$ antibiotic prescription in the year prior to the HELIUS study visit. Differences across ethnic groups were observed overall for any antibiotic prescription in both univariable $(p<0.001)$ and multivariable analysis $(p<0.001)$. In multivariable analysis, compared to Dutch individuals, first and second generation Ghanaian individuals and firstgeneration Moroccan individuals had significantly higher odds of receiving $\geq 1$ antibiotic prescription. Adding variables on antibiotic-related behavior and level of antibiotic use knowledge to the multivariable model did not change these associations.

Table 5 shows the results from the analysis on the association between ethnicity and total number of antibiotic prescriptions received in the year prior to the HELIUS study visit. Differences across ethnic groups were observed overall for the number of antibiotic prescriptions in both univariable and multivariable analysis (both $p=0.004$ ). First-generation African Surinamese and Turkish migrants had a significantly lower number of antibiotic prescriptions compared to individuals of Dutch origin. Only second-generation Ghanaian participants has more prescriptions compared to Dutch participants. Furthermore, female sex, diabetes mellitus, MI, malignant disorder, respiratory disease, eczema and worse perceived health were significantly associated with a higher number of antibiotic prescriptions.

Having a higher level of antibiotic knowledge was not significantly associated with the number of prescriptions when included in multivariable analysis $(p=0.446)$. No significant interactions between ethnicity and sex or education were observed. Finally, adjusting the association between ethnicity and antibiotic use for antibioticrelated behaviors did not change these associations.

\section{Discussion}

Our study shows that knowledge on the need to use antibiotics for treatment is lower among all ethnic minority groups compared to Dutch, with second generation ethnic minorities showing higher levels of knowledge compared to first generation migrants. We also observed ethnic differences in the use of antibiotics, with a higher proportion having received at least one prescription, but a lower mean number of antibiotic prescriptions among some ethnic minority groups compared to Dutch. The only ethnic group with a significantly higher number of 


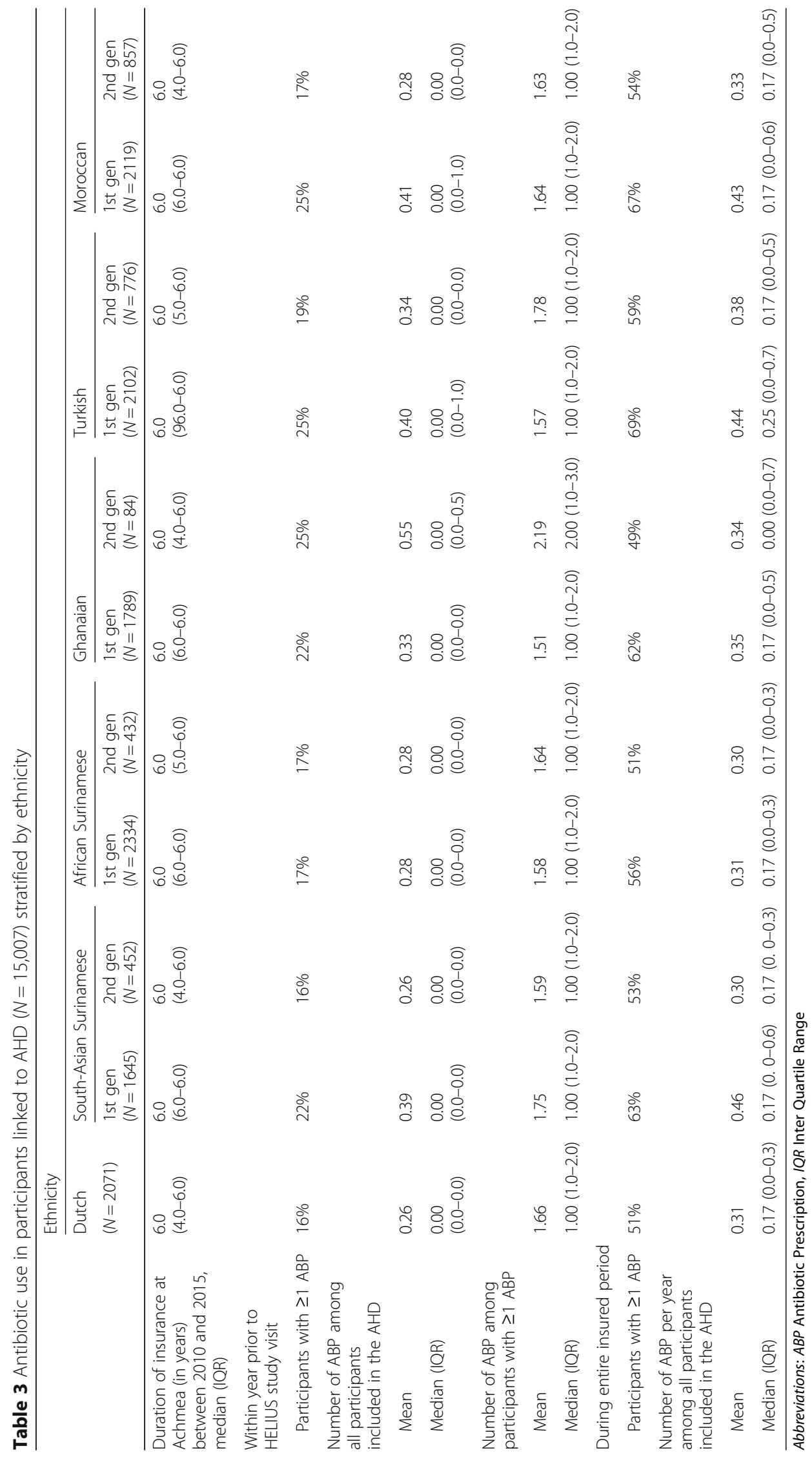


Table 4 Variables associated with having received $\geq 1$ antibiotic prescription in the year prior to HELIUS visit in participants linked to $\operatorname{AHD}(N=15,007)$ (logistic regression analysis)

\begin{tabular}{|c|c|c|c|c|c|c|c|c|c|}
\hline & \multicolumn{3}{|c|}{ Univariable } & \multicolumn{3}{|c|}{$\begin{array}{l}\text { Multivariable excluding } \\
\text { variables on antibiotic- } \\
\text { related behavior }\end{array}$} & \multicolumn{3}{|c|}{$\begin{array}{l}\text { Multivariable including } \\
\text { variables on antibiotic- } \\
\text { related behavior }\end{array}$} \\
\hline & OR & $(95 \% \mathrm{Cl})$ & $P$-values & $\mathrm{aOR}$ & $(95 \% \mathrm{Cl})$ & $P$-values & $\mathrm{aOR}$ & $(95 \% \mathrm{Cl})$ & $P$-values \\
\hline \multicolumn{10}{|l|}{ Sociodemographics } \\
\hline Ethnicity & & & $<.001$ & & & $<.001$ & & & .004 \\
\hline Dutch & Ref & & & Ref & & & Ref & & \\
\hline \multicolumn{10}{|l|}{ South-Asian Surinamese } \\
\hline 1st generation & 1.57 & $1.33-1.85$ & & 1.05 & $0.86-1.27$ & & 1.04 & $0.85-1.26$ & \\
\hline 2nd generation & 1.05 & $0.79-1.38$ & & 0.95 & $0.71-1.28$ & & 0.92 & $0.68-1.24$ & \\
\hline \multicolumn{10}{|l|}{ African Surinamese } \\
\hline 1st generation & 1.15 & $0.98-1.35$ & & 0.89 & $0.75-1.07$ & & 0.88 & $0.73-1.05$ & \\
\hline 2nd generation & 1.14 & $0.87-1.50$ & & 1.02 & $0.76-1.36$ & & 0.96 & $0.71-1.29$ & \\
\hline \multicolumn{10}{|l|}{ Ghanaian } \\
\hline 1st generation & 1.53 & $1.30-1.81$ & & 1.38 & $1.14-1.68$ & & 1.28 & $1.05-1.56$ & \\
\hline 2nd generation & 1.81 & $1.09-3.01$ & & 1.92 & $1.12-3.27$ & & 1.64 & $0.94-2.87$ & \\
\hline \multicolumn{10}{|l|}{ Turkish } \\
\hline 1st generation & 1.84 & $1.58-2.15$ & & 1.07 & $0.88-1.31$ & & 1.00 & $0.82-1.22$ & \\
\hline 2nd generation & 1.27 & $1.02-1.57$ & & 1.02 & $0.80-1.30$ & & 1.01 & $0.79-1.29$ & \\
\hline \multicolumn{10}{|l|}{ Moroccan } \\
\hline 1st generation & 1.81 & $1.55-2.11$ & & 1.22 & $1.00-1.49$ & & 1.15 & $0.94-1.41$ & \\
\hline 2nd generation & 1.14 & $0.92-1.41$ & & 0.93 & $0.73-1.19$ & & 0.89 & $0.69-1.14$ & \\
\hline Female sex & 1.91 & $1.75-2.08$ & $<.001$ & 1.77 & $1.60-1.95$ & $<.001$ & 1.70 & $1.54-1.88$ & $<.001$ \\
\hline Age & & & $<.001$ & & & & & & \\
\hline$<25$ years & Ref & & & & & & & & \\
\hline $25-34$ years & 1.04 & $0.87-1.24$ & & & & & & & \\
\hline $35-44$ years & 1.28 & $1.09-1.50$ & & & & & & & \\
\hline $45-54$ years & 1.34 & $1.15-1.56$ & & & & & & & \\
\hline $55-64$ years & 1.42 & $1.21-1.66$ & & & & & & & \\
\hline$\geq 65$ years & 1.59 & $1.29-1.96$ & & & & & & & \\
\hline Educational level & & & $<.001$ & & & .005 & & & .001 \\
\hline Unknown & Ref & & & Ref & & & Ref & & \\
\hline No school/elementary school & 1.41 & $0.95-2.09$ & & 1.55 & $0.93-2.58$ & & 1.55 & $0.87-2.75$ & \\
\hline Lower vocational/lower secondary school & 1.05 & $0.71-1.55$ & & 1.50 & $0.90-2.50$ & & 1.47 & $0.83-2.60$ & \\
\hline Intermediate vocational/ intermediate secondary school & 0.93 & $0.63-1.39$ & & 1.43 & $0.86-2.39$ & & 1.38 & $0.78-2.44$ & \\
\hline Higher vocational/university & 0.66 & $0.44-0.99$ & & 1.18 & $0.71-1.99$ & & 1.12 & $0.63-2 . .00$ & \\
\hline Marital status & & & $<.001$ & & & & & & \\
\hline Married/registered partnership & Ref & & & & & & & & \\
\hline Cohabiting & 0.66 & $0.56-0.77$ & & & & & & & \\
\hline Unmarried/never married & 0.78 & $0.71-0.86$ & & & & & & & \\
\hline Divorced/separated & 1.14 & $1.02-1.27$ & & & & & & & \\
\hline Widow/widower & 1.26 & $0.98-1.64$ & & & & & & & \\
\hline \multicolumn{10}{|l|}{ Health status } \\
\hline \multicolumn{10}{|l|}{ Self-reported medical conditions (previous 12 months) } \\
\hline Diabetes mellitus & 1.75 & $1.56-1.96$ & $<.001$ & 1.29 & $1.13-1.47$ & $<.001$ & 1.30 & $1.13-1.48$ & $<.001$ \\
\hline
\end{tabular}


Table 4 Variables associated with having received $\geq 1$ antibiotic prescription in the year prior to HELIUS visit in participants linked to AHD $(N=15,007)$ (logistic regression analysis) (Continued)

\begin{tabular}{|c|c|c|c|c|c|c|c|c|c|}
\hline & \multicolumn{3}{|c|}{ Univariable } & \multicolumn{3}{|c|}{$\begin{array}{l}\text { Multivariable excluding } \\
\text { variables on antibiotic- } \\
\text { related behavior }\end{array}$} & \multicolumn{3}{|c|}{$\begin{array}{l}\text { Multivariable including } \\
\text { variables on antibiotic- } \\
\text { related behavior }\end{array}$} \\
\hline & OR & $(95 \% \mathrm{Cl})$ & $P$-values & $\mathrm{aOR}$ & $(95 \% \mathrm{Cl})$ & $P$-values & $\mathrm{aOR}$ & $(95 \% \mathrm{Cl})$ & $P$-values \\
\hline CVA/one-sided loss of bodily function $\leq 1$ day & 1.38 & $1.18-1.62$ & $<.001$ & & & & & & \\
\hline Ml incl. $\geq$ half hour chest pain or dotter/bypass operation & 1.78 & $1.60-1.98$ & $<.001$ & 1.28 & $1.13-1.45$ & $<.001$ & 1.24 & $1.09-1.40$ & .001 \\
\hline Severe heart condition & 1.45 & $1.18-1.79$ & .001 & & & & & & \\
\hline Malignant disorder & 1.93 & $1.52-2.47$ & $<.001$ & 1.33 & $1.02-1.74$ & .037 & & & \\
\hline Severe or chronic fatigue & 1.86 & $1.71-2.02$ & $<.001$ & 1.20 & $1.08-1.33$ & .001 & 1.16 & $1.04-1.29$ & .008 \\
\hline High blood pressure & 1.36 & $1.25-1.49$ & $<.001$ & & & & & & \\
\hline Artery stenosis & 1.46 & $1.25-1.70$ & $<.001$ & 0.80 & $0.67-0.96$ & .014 & 0.77 & $0.64-0.92$ & .004 \\
\hline Respiratory diseases & 2.19 & $1.96-2.44$ & $<.001$ & 1.66 & $1.47-1.87$ & $<.001$ & 1.59 & $1.41-1.81$ & $<.001$ \\
\hline Serious/persistent intestinal disorders & 1.87 & $1.64-2.12$ & $<.001$ & 1.24 & $1.07-1.43$ & .004 & 1.22 & $1.05-1.41$ & .009 \\
\hline Psoriasis & 1.28 & $1.05-1.56$ & .015 & & & & & & \\
\hline (Chronic) eczema & 1.30 & $1.15-1.47$ & $<.001$ & & & & & & \\
\hline Incontinence & 2.08 & $1.85-2.35$ & $<.001$ & 1.32 & $1.15-1.52$ & $<.001$ & 1.32 & $1.15-1.52$ & $<.001$ \\
\hline Body Mass Index & & & $<.001$ & & & & & & \\
\hline$<18.5$ & Ref & & & & & & & & \\
\hline $18.5-25$ & 1.03 & $0.72-1.46$ & & & & & & & \\
\hline $25-30$ & 1.24 & $0.87-1.76$ & & & & & & & \\
\hline $30-40$ & 1.64 & $1.15-2.33$ & & & & & & & \\
\hline$\geq 40$ & 1.97 & $1.31-2.97$ & & & & & & & \\
\hline Smoking & & & .184 & & & $<.001$ & & & .003 \\
\hline Yes & Ref & & & Ref & & & Ref & & \\
\hline No, never & 0.99 & $0.90-1.09$ & & 0.78 & $0.69-0.87$ & & 0.82 & $0.72-0.92$ & \\
\hline No, but ever & 0.90 & $0.80-1.02$ & & 0.91 & $0.79-1.04$ & & 0.93 & $0.81-1.07$ & \\
\hline Alcohol usage & & & $<.001$ & & & .017 & & & .012 \\
\hline Never & Ref & & & Ref & & & Ref & & \\
\hline Not in previous 12 months & 0.88 & $0.77-1.02$ & & 0.96 & $0.82-1.12$ & & 0.95 & $0.81-1.12$ & \\
\hline Monthly or less & 0.68 & $0.61-0.77$ & & 0.83 & $0.72-0.95$ & & 0.82 & $0.71-0.94$ & \\
\hline 2-4 times per month & 0.73 & $0.64-0.83$ & & 0.93 & $0.79-1.09$ & & 0.92 & $0.78-1.08$ & \\
\hline 2-3 times per week & 0.64 & $0.55-0.75$ & & 0.92 & $0.76-1.10$ & & 0.89 & $0.74-1.08$ & \\
\hline$\geq 4$ times per week & 0.48 & $0.39-0.58$ & & 0.70 & $0.55-0.88$ & & 0.68 & $0.54-0.86$ & \\
\hline Difficulty with Dutch language & & & $<.001$ & & & & & & \\
\hline No & Ref & & & & & & & & \\
\hline Yes & 1.32 & $1.21-1.43$ & & & & & & & \\
\hline Not applicable & 0.80 & $0.71-0.91$ & & & & & & & \\
\hline Perceived health & & & $<.001$ & & & $<.001$ & & & .002 \\
\hline Excellent & Ref & & & Ref & & & Ref & & \\
\hline Very good & 1.09 & $0.86-1.37$ & & 1.05 & $0.83-1.34$ & & 1.03 & $0.80-1.31$ & \\
\hline Good & 1.55 & $1.27-1.90$ & & 1.22 & $0.99-1.51$ & & 1.20 & $0.97-1.49$ & \\
\hline Mediocre & 2.47 & $2.01-3.04$ & & 1.37 & $1.09-1.72$ & & 1.32 & $1.05-1.67$ & \\
\hline Bad & 3.85 & $3.02-4.91$ & & 1.69 & $1.28-2.23$ & & 1.59 & $1.20-2.11$ & \\
\hline \multicolumn{10}{|l|}{ Antibiotic-related behavior } \\
\hline Higher antibiotic knowledge & & & $<.001$ & & & & & & \\
\hline
\end{tabular}


Table 4 Variables associated with having received $\geq 1$ antibiotic prescription in the year prior to HELIUS visit in participants linked to AHD $(N=15,007)$ (logistic regression analysis) (Continued)

\begin{tabular}{|c|c|c|c|c|c|c|c|c|c|}
\hline & \multicolumn{3}{|c|}{ Univariable } & \multicolumn{3}{|c|}{$\begin{array}{l}\text { Multivariable excluding } \\
\text { variables on antibiotic- } \\
\text { related behavior }\end{array}$} & \multicolumn{3}{|c|}{$\begin{array}{l}\text { Multivariable including } \\
\text { variables on antibiotic- } \\
\text { related behavior }\end{array}$} \\
\hline & OR & $(95 \% \mathrm{Cl})$ & $P$-values & $\mathrm{aOR}$ & $(95 \% \mathrm{Cl})$ & $P$-values & $\mathrm{aOR}$ & $(95 \% \mathrm{Cl})$ & $P$-values \\
\hline No & Ref & & & & & & & & \\
\hline Yes & 0.77 & $0.71-0.84$ & & & & & & & \\
\hline Ever asked GP for antibiotics & & & $<.001$ & & & & & & $<.001$ \\
\hline No, never & Ref & & & & & & Ref & & \\
\hline Yes, regularly & 4.72 & $3.59-6.21$ & & & & & 3.07 & $2.28-4.14$ & \\
\hline Yes, occasionally & 2.42 & $2.20-2.66$ & & & & & 2.11 & $1.91-2.34$ & \\
\hline Did not finish treatment & & & $<.001$ & & & & & & $<.001$ \\
\hline Always finished or no antibiotics & Ref & & & & & & Ref & & \\
\hline Yes, regularly & 2.70 & $2.02-3.62$ & & & & & 1.80 & $1.29-2.50$ & \\
\hline Yes, occasionally & 1.62 & $1.45-1.83$ & & & & & 1.32 & $1.16-1.50$ & \\
\hline
\end{tabular}

Abbreviations: OR Odds Ratio, aOR adjusted Odds Ratio, Cl Confidence Interval, CVA Cerebro Vascular Accident, MI Myocardial infarction, GP General Practitioner

antibiotic prescriptions was second generation Ghanaian participants. Furthermore, we showed that a lower level of antibiotic knowledge was not associated with receiving antibiotics or average number of antibiotic prescriptions, and that ethnic differences in antibiotic use therefore cannot be explained by level of knowledge on antibiotics.

A previous study in Dutch primary care centres demonstrated higher use of antibiotics among firstgeneration migrants from Turkey, Morocco, Surinam or the Antilles compared to Dutch, after adjustment for age, sex, education, presence of chronic diseases, and smoking [3]. We found that the odds of having $\geq 1$ antibiotic prescription was higher in some ethnic groups in unadjusted analysis, but after adjusting for several variables including medical conditions, the odds were significantly higher among Ghanaian and first-generation Moroccan participants only. In contrast, in our analyses on the number of antibiotic prescriptions as an outcome, only second-generation Ghanaian migrants were at higher risk of receiving a higher number of prescriptions compared to Dutch participants. For all other ethnic groups, no evidence of a higher risk for more frequent prescriptions was found, while even a lower number was present for first-generation African Suriname and Turkish participants. To the best of our knowledge, no other studies have evaluated the variation in level of antibiotic knowledge and antibiotic use between ethnic groups and thus our findings need to be confirmed. Notably, our findings on antibiotic prescriptions and ethnicity are in line with a large retrospective cohort study performed in pediatric emergency departments in the United States [15]. This study also looked at the association between ethnicity and antibiotic prescribing, showing that other ethnic groups received less antibiotics for viral infections than non-Hispanic white children.

Lower odds for higher level of antibiotic use knowledge were also seen among individuals who regularly or occasionally requested antibiotics from their GP or who regularly or occasionally did not finish treatment. These findings suggest that improving antibiotic knowledge might decrease the number of requests for antibiotics in primary care and improve appropriate use.

Our study has several strengths. First, the HELIUS study consists of a large number of participants from major ethnic groups living in the same city, with representation from all socioeconomic levels. Second, all outcomes and determinants were measured using the same methodology across all ethnic groups and HELIUS used translated questionnaires and had ethnically-matched interviewers and research assistants to provide assistance during data collection. These procedures enhance the comparability between ethnic groups. Another major strength of the current study is that HELIUS data could be linked to data from a health insurance register covering the majority $(77.7 \%)$ of the study population.

Our study has also limitations. First, although HELIUS participants were recruited via an ethnicity-stratified random selection of the municipal registry of Amsterdam, the response rate for HELIUS study was $28 \%$ and there may be selection bias [8]. However, analysis from a previous HELIUS study have shown that participants are not exceedingly different from nonrespondents regarding sociodemographic variables [8]. Second, we did not take into account the use of antibiotics purchased over the counter in the home country of participants [6, 16-18], and we might therefore have underestimated antibiotic use in non-Dutch ethnic 
Table 5 Variables associated with number of antibiotic prescriptions in participants linked to ADH $(N=15,007)$ (zero-inflated negative binomial regression analysis)

\begin{tabular}{|c|c|c|c|c|c|c|c|c|c|}
\hline & \multicolumn{3}{|c|}{ Univariable $^{a}$} & \multicolumn{3}{|c|}{$\begin{array}{l}\text { Multivariable excluding } \\
\text { variables on antibiotic- } \\
\text { related behavior }\end{array}$} & \multicolumn{3}{|c|}{$\begin{array}{l}\text { Multivariable including } \\
\text { variables on antibiotic- } \\
\text { related behavior }\end{array}$} \\
\hline & IRR & $(95 \% \mathrm{Cl})$ & $P$-values & IRR & $(95 \% \mathrm{Cl})$ & $P$-values & $\mathrm{IRR}$ & $(95 \% \mathrm{Cl})$ & $P$-values \\
\hline \multicolumn{10}{|l|}{ Sociodemographics } \\
\hline Ethnicity & & & .004 & & & .004 & & & .001 \\
\hline Dutch & Ref & & & Ref & & & Ref & & \\
\hline \multicolumn{10}{|l|}{ South-Asian Surinamese } \\
\hline 1st generation & 1.06 & $0.85-1.31$ & & 0.86 & $0.68-1.10$ & & 1.02 & $0.82-1.28$ & \\
\hline 2nd generation & 0.82 & $0.55-1.21$ & & 0.94 & $0.61-1.44$ & & 0.94 & $0.64-1.36$ & \\
\hline \multicolumn{10}{|l|}{ African Surinamese } \\
\hline 1st generation & 0.79 & $0.64-0.99$ & & 0.73 & $0.58-0.93$ & & 0.81 & $0.65-1.00$ & \\
\hline 2nd generation & 0.83 & $0.57-1.22$ & & 0.90 & $0.59-1.36$ & & 0.93 & $0.65-1.33$ & \\
\hline \multicolumn{10}{|l|}{ Ghanaian } \\
\hline 1st generation & 0.75 & $0.60-0.94$ & & 0.77 & $0.60-1.00$ & & 0.81 & $0.65-1.02$ & \\
\hline 2nd generation & 1.66 & $0.89-3.11$ & & 2.09 & $1.06-4.12$ & & 2.70 & $1.47-4.94$ & \\
\hline \multicolumn{10}{|l|}{ Turkish } \\
\hline 1st generation & 0.85 & $0.69-1.04$ & & 0.74 & $0.59-0.92$ & & 0.79 & $0.64-0.97$ & \\
\hline 2nd generation & 1.14 & $0.87-1.51$ & & 1.14 & $0.84-1.53$ & & 1.10 & $0.84-1.44$ & \\
\hline \multicolumn{10}{|l|}{ Moroccan } \\
\hline 1st generation & 0.99 & $0.81-1.21$ & & 0.89 & $0.70-1.11$ & & 0.89 & $0.72-1.10$ & \\
\hline 2nd generation & 0.84 & $0.63-1.13$ & & 0.92 & $0.67-1.27$ & & 1.02 & $0.76-1.37$ & \\
\hline Female sex & 1.36 & $1.20-1.54$ & $<.001$ & 1.35 & $1.18-1.54$ & $<.001$ & 1.29 & $1.15-1.46$ & $<.001$ \\
\hline Age & & & .023 & & & & & & \\
\hline$<25$ years & Ref & & & & & & & & \\
\hline $25-34$ years & 1.09 & $0.91-1.30$ & & & & & & & \\
\hline $35-44$ years & 1.16 & $0.98-1.38$ & & & & & & & \\
\hline $45-54$ years & 1.09 & $0.92-1.29$ & & & & & & & \\
\hline $55-64$ years & 1.14 & $0.95-1.36$ & & & & & & & \\
\hline$\geq 65$ years & 1.44 & $1.16-1.80$ & & & & & & & \\
\hline Educational level & & & .096 & & & & & & \\
\hline Unknown & Ref & & & & & & & & \\
\hline No school/elementary school & 1.74 & $0.86-3.52$ & & & & & & & \\
\hline Lower vocational/lower secondary school & 1.74 & $0.86-3.52$ & & & & & & & \\
\hline Intermediate vocational/ intermediate secondary school & 1.57 & $0.77-3.17$ & & & & & & & \\
\hline Higher vocational/university & 1.45 & $0.71-2.96$ & & & & & & & \\
\hline Marital status & & & .212 & & & & & & \\
\hline Married/registered partnership & Ref & & & & & & & & \\
\hline Cohabiting & 0.85 & $0.72-1.00$ & & & & & & & \\
\hline Unmarried/never married & 0.99 & $0.89-1.10$ & & & & & & & \\
\hline Divorced/separated & 1.01 & $0.90-1.13$ & & & & & & & \\
\hline Widow/widower & 1.14 & $0.90-1.45$ & & & & & & & \\
\hline
\end{tabular}

Health status

Self-reported medical conditions (previous 12 months)

Diabetes mellitus 
Table 5 Variables associated with number of antibiotic prescriptions in participants linked to ADH $(N=15,007)$ (zero-inflated negative binomial regression analysis) (Continued)

\begin{tabular}{|c|c|c|c|c|c|c|c|c|c|}
\hline & \multicolumn{3}{|c|}{ Univariable $^{a}$} & \multicolumn{3}{|c|}{$\begin{array}{l}\text { Multivariable excluding } \\
\text { variables on antibiotic- } \\
\text { related behavior }\end{array}$} & \multicolumn{3}{|c|}{$\begin{array}{l}\text { Multivariable including } \\
\text { variables on antibiotic- } \\
\text { related behavior }\end{array}$} \\
\hline & IRR & $(95 \% \mathrm{Cl})$ & $P$-values & IRR & $(95 \% \mathrm{Cl})$ & $P$-values & IRR & $(95 \% \mathrm{Cl})$ & $P$-values \\
\hline CVA/one-sided loss of bodily function $\leq 1$ day & 1.10 & $0.95-1.29$ & .208 & & & & & & \\
\hline MI incl. Zhalf hour chest pain or dotter/bypass operation & 1.35 & $1.19-1.54$ & $<.001$ & 1.22 & $1.06-1.41$ & .005 & & & \\
\hline Severe heart condition & 1.26 & $1.04-1.52$ & .019 & & & & & & \\
\hline Malignant disorder & 1.91 & $1.47-2.48$ & $<.001$ & 1.60 & $1.21-2.12$ & .001 & 1.60 & $1.28-2.00$ & \\
\hline Severe or chronic fatigue & 1.29 & $1.15-1.44$ & $<.001$ & & & & & & \\
\hline High blood pressure & 1.14 & $1.04-1.25$ & .006 & & & & & & \\
\hline Artery stenosis & 1.30 & $1.08-1.57$ & .005 & & & & & & \\
\hline Respiratory diseases & 1.50 & $1.32-1.71$ & $<.001$ & 1.34 & $1.16-1.54$ & $<.001$ & 1.29 & $1.13-1.47$ & $<.001$ \\
\hline Serious/persistent intestinal disorders & 1.24 & $1.07-1.44$ & .004 & & & & & & \\
\hline Psoriasis & 1.08 & $0.90-1.31$ & .406 & & & & & & \\
\hline (Chronic) eczema & 1.21 & $1.07-1.37$ & .002 & 1.14 & $1.00-1.29$ & .042 & & & \\
\hline Incontinence & 1.31 & $1.13-1.50$ & $<.001$ & & & & & & \\
\hline Body Mass Index & & & .736 & & & & & & \\
\hline$<18.5$ & Ref & & & & & & & & \\
\hline $18.5-25$ & 0.98 & $0.70-1.37$ & & & & & & & \\
\hline $25-30$ & 0.98 & $0.70-1.37$ & & & & & & & \\
\hline $30-40$ & 1.04 & $0.74-1.46$ & & & & & & & \\
\hline$\geq 40$ & 1.06 & $0.71-1.57$ & & & & & & & \\
\hline Smoking & & & .099 & & & & & & \\
\hline Yes & Ref & & & & & & & & \\
\hline No, never & 1.13 & $1.00-1.29$ & & & & & & & \\
\hline No, but ever & 1.02 & $0.87-1.21$ & & & & & & & \\
\hline Alcohol usage & & & .075 & & & & & & \\
\hline Never & Ref & & & & & & & & \\
\hline Not in previous 12 months & 0.93 & $0.77-1.13$ & & & & & & & \\
\hline Monthly or less & 0.94 & $0.80-1.11$ & & & & & & & \\
\hline 2-4 times per month & 1.01 & $0.85-1.20$ & & & & & & & \\
\hline 2-3 times per week & 0.73 & $0.58-0.91$ & & & & & & & \\
\hline$\geq 4$ times per week & 0.81 & $0.61-1.08$ & & & & & & & \\
\hline Difficulty with Dutch language & & & .320 & & & & & & \\
\hline No & Ref & & & & & & & & \\
\hline Yes & 0.94 & $0.86-1.04$ & & & & & & & \\
\hline Not applicable & 1.05 & $0.88-1.25$ & & & & & & & \\
\hline Perceived health & & & $<.001$ & & & .001 & & & $<.001$ \\
\hline Excellent & Ref & & & Ref & & & Ref & & \\
\hline Very good & 0.98 & $0.68-1.42$ & & 1.03 & $0.71-1.49$ & & 0.99 & $0.70-1.39$ & \\
\hline Good & 1.29 & $0.94-1.77$ & & 1.19 & $0.85-1.65$ & & 1.17 & $0.86-1.59$ & \\
\hline Mediocre & 1.73 & $1.25-2.39$ & & 1.40 & $1.00-1.97$ & & 1.44 & $1.05-1.97$ & \\
\hline Bad & 2.30 & $1.62-3.26$ & & 1.71 & $1.17-2.49$ & & 1.72 & $1.22-2.43$ & \\
\hline \multicolumn{10}{|l|}{ Antibiotic-related behavior } \\
\hline Higher antibiotic knowledge & & & .054 & & & & & & \\
\hline
\end{tabular}


Table 5 Variables associated with number of antibiotic prescriptions in participants linked to ADH $(N=15,007)$ (zero-inflated negative binomial regression analysis) (Continued)

\begin{tabular}{|c|c|c|c|c|c|c|c|c|c|}
\hline & \multicolumn{3}{|c|}{ Univariable $^{a}$} & \multicolumn{3}{|c|}{$\begin{array}{l}\text { Multivariable excluding } \\
\text { variables on antibiotic- } \\
\text { related behavior }\end{array}$} & \multicolumn{3}{|c|}{$\begin{array}{l}\text { Multivariable including } \\
\text { variables on antibiotic- } \\
\text { related behavior }\end{array}$} \\
\hline & IRR & $(95 \% \mathrm{Cl})$ & $P$-values & $\overline{I R R}$ & $(95 \% \mathrm{Cl})$ & $P$-values & $\overline{\mathrm{IRR}}$ & $(95 \% \mathrm{Cl})$ & $P$-values \\
\hline No & Ref & & & & & & & & \\
\hline Yes & 0.92 & $0.85-1.00$ & & & & & & & \\
\hline Ever asked GP for antibiotics & & & $<.001$ & & & & & & $<.001$ \\
\hline No, never & Ref & & & & & & Ref & & \\
\hline Yes, regularly & 2.96 & $2.34-3.73$ & & & & & 1.87 & $1.45-2.41$ & \\
\hline Yes, occasionally & 1.75 & $1.59-1.92$ & & & & & 1.15 & $1.06-1.30$ & \\
\hline Did not finish treatment & & & $<.001$ & & & & & & \\
\hline Always finished or no antibiotics & Ref & & & & & & & & \\
\hline Yes, regularly & 1.50 & $1.12-2.00$ & & & & & & & \\
\hline Yes, occasionally & 1.32 & $1.18-1.48$ & & & & & & & \\
\hline
\end{tabular}

Abbreviations: IRR Incidence Risk Ratio, CI Confidence Interval, CVA Cerebro Vascular Accident, MI Myocardial infarction, GP General Practitioner

${ }^{a}$ Accounts for zero-inflated distribution

groups. As a recent HELIUS study found that Dutch people of Turkish or Moroccan origin were more likely to use healthcare in the Netherlands as well as their country of origin [19], underestimation of antibiotic use in non-Dutch ethnic groups seems unlikely. Third, since several characteristics, such as education level and medical conditions, of HELIUS participants insured at Achmea differed from those insured elsewhere, selection bias could have been introduced in analysis on antibiotic use. This difference could be due to the fact that the City of Amsterdam provided health insurance discounts with Achmea for low-income individuals. These differences were corrected for during multivariable analyses to the most possible extent. Fourth, the variable 'ever asked GP for antibiotics' does not discriminate between appropriate or inappropriate requests for antibiotics and misclassification might have occurred. However, this variable gives some information on participants' attitudes towards antibiotic use. Furthermore, due to privacy restrictions, we were unable to include indication for antibiotic therapy and duration of antibiotic use as additional indices for antibiotic use (apart from the number of antibiotics prescribed). Moreover, since this was a cross-sectional study, we were unable to model antibiotic knowledge with future antibiotic prescriptions. Further research should examine the association of antibiotic knowledge with future antibiotic prescriptions. Finally, we are unable to determine if individuals were more demanding towards their GP or if their GPs were more lenient in prescribing antibiotics during illness $[4,5]$. Neither completing antibiotic therapy, assessed by pill count, nor duration of antibiotic use could be taken into account as these data were not available.

\section{Conclusions}

To our knowledge, this study is the first to examine ethnic disparities in level of antibiotic knowledge and use in a large population-based sample among adults with different ethnic backgrounds. Health policy makers and healthcare professionals are increasingly developing interventions to improve the quality of antibiotic use, which is needed to help contain antimicrobial resistance. Targeted campaigns can be considered, for instance, during the annual European Antibiotic Awareness Day, since this event addresses improvement in the quality of antibiotic use to the general public [20]. Still, this study shows that a lower level of antibiotic knowledge is not necessarily linked to higher antibiotic usage, indicating that interventions aimed at improving knowledge alone might be insufficient to reduce antibiotic use. Nevertheless, the underlying reasons for these findings need further evaluation.

\section{Supplementary information}

Supplementary information accompanies this paper at https://doi.org/10. 1186/s13756-019-0636-x

Additional file 1. Supplementary Methods.

Additional file 2: Table S1. Characteristics of participants not linked versus linked to the Achmea Health Database.

\section{Abbreviations}

AHD: Achmea Health Database; alRR: adjusted Incidence Risk Ratio; aOR: adjusted Odds Ratio; BMI: Body Mass Index; CVA: Cerebrovascular Accident; GP: General Practitioner; HELIUS: Healthy life in an Urban Setting; IQR: Interquartile Range; IRR: Incidence Risk Ratio; MI: Myocardial Infarction; OR: Odds Ratio; WHO: World Health Organization

\section{Acknowledgements}

The authors would like to acknowledge the HELIUS participants for their contribution; the HELIUS team for data collection and management. 


\section{Authors' contributions}

$E S, M S, S G, M P$ and JP were involved in the conception and design of the study. ES, EvD and MS were involved in the acquisition of data. EvD and $A B$ analyzed the data. ES and EvD wrote the manuscript. ES, EvD, AB, MS, SG, MP and JP were major contributors in revising the manuscript. All authors read and approved the final manuscript.

\section{Funding}

The HELIUS study is conducted by the Academic Medical Center Amsterdam and the Public Health Service of Amsterdam. Both organizations provided core support for HELIUS. The HELIUS study is also funded by the Dutch Heart Foundation (2010 T084), the Netherlands Organization for Health Research and Development (ZonMw) (200500003), the European Union (FP-7) (278901), and the European Fund for the Integration of non-EU immigrants (EIF) (2013EIF013). The authors were independent from funders and had ful access to all data. The decision to submit for publication was uninfluenced by the funders. The authors of this study take full responsibility for the integrity of the data and the accuracy of the data analysis.

\section{Availability of data and materials}

The datasets used in the current study are available from the corresponding author on request, contingent on approval from the HELIUS scientific committee.

\section{Ethics approval and consent to participate}

The HELIUS study is conducted in accordance with the Declaration of Helsinki and has been approved by the AMC Ethical Review Board. All participants provided written informed consent.

\section{Consent for publication}

Not applicable.

\section{Competing interests}

The authors declare that they have no competing interests.

\section{Author details}

'Department of Internal Medicine, Division of Infectious Diseases, Amsterdam UMC, University of Amsterdam, Meibergdreef 9, 1105, AZ, Amsterdam, The Netherlands. ${ }^{2}$ Department of Infectious Diseases, Public Health Service Amsterdam, Nieuwe Achtergracht 100, 1018, WT, Amsterdam, The Netherlands. ${ }^{3}$ INSERM, Sorbonne Université, Institut Pierre Louis d'Épidémiologie et de Santé Publique, Paris, France. ${ }^{4}$ Department of Public Health, Amsterdam UMC, University of Amsterdam, Amsterdam Public Health Research Institute, Amsterdam, The Netherlands. ${ }^{5}$ Department of Clinical Epidemiology, Biostatistics and Bioinformatics, Amsterdam UMC, University of Amsterdam, Amsterdam Public Health Research Institute, Amsterdam, The Netherlands.

Received: 31 July 2019 Accepted: 24 October 2019

Published online: 06 December 2019

\section{References}

1. WHO. Antimicrobial resistance: global report on surveillance 2014; 2014. p. 257.

2. Nellums LB, Thompson H, Holmes A, Castro-Sanchez E, Otter JA, Norredam $M$, et al. Antimicrobial resistance among migrants in Europe: a systematic review and meta-analysis. Lancet Infect Dis. 2018;18(7):796-811.

3. Hogenhuis CC, Grigoryan L, Numans MM, Verheij TJ. Differences in antibiotic treatment and utilization of diagnostic tests in Dutch primary care between natives and non-western immigrants. Eur J Gen Pract. 2010;16(3):143-7.

4. Grigoryan L, Burgerhof JG, Degener JE, Deschepper R, Lundborg CS, Monnet DL, et al. Determinants of self-medication with antibiotics in Europe: the impact of beliefs, country wealth and the healthcare system. $J$ Antimicrob Chemother. 2008;61(5):1172-9.

5. Grigoryan L, Burgerhof JG, Degener JE, Deschepper R, Lundborg CS, Monnet DL, et al. Attitudes, beliefs and knowledge concerning antibiotic use and self-medication: a comparative European study. Pharmacoepidemiol Drug Saf. 2007;16(11):1234-43.

6. Norris P, Ng LF, Kershaw V, Hanna F, Wong A, Talekar M, et al. Knowledge and reported use of antibiotics amongst immigrant ethnic groups in New Zealand. J Immigr Minor Health. 2010;12(1):107-12.
7. Deschepper R, Grigoryan L, Lundborg CS, Hofstede G, Cohen J, Kelen GV, et al. Are cultural dimensions relevant for explaining cross-national differences in antibiotic use in Europe? BMC Health Serv Res. 2008;8:123.

8. Snijder MB, Galenkamp H, Prins M, Derks EM, Peters RJG, Zwinderman AH, et al. Cohort profile: the Healthy life in an urban setting (HELIUS) study in Amsterdam, The Netherlands. BMJ Open. 2017;7(12):e017873.

9. Stronks K, Snijder MB, Peters RJ, Prins M, Schene AH, Zwinderman AH. Unravelling the impact of ethnicity on health in Europe: the HELIUS study. BMC Public Health. 2013;13:402.

10. StatLine. Population key figures 2019. https:/opendata.cbs.nl/statline/\#/CBS/ nl/dataset/37296ned/table?ts=156888214600.

11. StatLine. Population data; age, migration background, gender and region. 2019. https://opendata.cbs.nl/statline/\#/CBS/nl/dataset/37713/table?ts=156 8882255075.

12. Stronks K, Kulu-Glasgow I, Agyemang C. The utility of 'country of birth' for the classification of ethnic groups in health research: the Dutch experience. Ethn Health. 2009:14(3):255-69.

13. Data Protection Authority. Ministry of Health, Welfare and Sport. https:// autoriteitpersoonsgegevens.nl/en.

14. Special Eurobarometer 338: Antimicrobial Resistance (2010). http://ec. europa.eu/public_opinion/archives/eb_special_339_320_en.htm\#338.

15. Goyal MK, Johnson TJ, Chamberlain JM, Casper TC, Simmons T, Alessandrini EA, et al. Racial and Ethnic Differences in Antibiotic Use for Viral Illness in Emergency Departments. Pediatrics. 2017;140(2):e20170203 (4).

16. Lindenmeyer A, Redwood S, Griffith L, Ahmed S, Phillimore J. Recent migrants' perspectives on antibiotic use and prescribing in primary care: a qualitative study. Br J Gen Pract. 2016;66(652):e802-e9.

17. Hu J, Wang Z. Non-prescribed antibiotic use and general practitioner service utilisation among Chinese migrants in Australia. Aust J Prim Health. 2016; 22(5):434-9.

18. Hu J, Wang Z. In-home antibiotic storage among Australian Chinese migrants. Int J Infect Dis. 2014;26:103-6.

19. Sekercan A, Snijder MB, Peters RJG, Stronks K. Is healthcare consumption in the country of origin among Moroccan and Turkish migrants of older age (55+) associated with less use of care in the Netherlands? Tijdschr Gerontol Geriatr. 2018;49(6):253-62.

20. European Centre for Disease Prevention and Control. Antimicrobial consumption. In: ECDC, editor. Annual epidemiological report for 2016. Stockholm: ECDC; 2018. https:/ecdc.europa.eu/en/publications-data/ antimicrobial-consumption-annual-epidemiological-report-2016.

\section{Publisher's Note}

Springer Nature remains neutral with regard to jurisdictional claims in published maps and institutional affiliations.

Ready to submit your research? Choose BMC and benefit from:

- fast, convenient online submission

- thorough peer review by experienced researchers in your field

- rapid publication on acceptance

- support for research data, including large and complex data types

- gold Open Access which fosters wider collaboration and increased citations

- maximum visibility for your research: over $100 \mathrm{M}$ website views per year

At BMC, research is always in progress.

Learn more biomedcentral.com/submissions 